\title{
Transient Crack Propagation in Asymmetric Cruciform Paths
}

\author{
By \\ H. G. Georgiadis, Athens, Greece \\ With 4 Figures \\ (Received April 30, 1986; revised October 31, 1986)
}

\begin{abstract}
Summary
The problem of two cracks emanating from the same origin and propagating asymmetrically at different velocities in an elastic and isotropie solid is treated in this paper. An unbounded and otherwise undisturbed medium and a constant anti-plane loading at infinity were assumed. Techniques of self-similar elastodynamics were utilized in conjunction with analytic-function theory. Since a closed-form solution of such a problem is impossible we relied in the last steps of the procedure upon numerical analysis.
\end{abstract}

\section{Introduction}

Dynamic fracture is a subject which continues to concentrate the interest of workers of applied mechanics. In this realm also belongs the present work. Relevant references which review the most basic theoretical studies in this field were published by Achenbach [1], [2], Freund [3] and Sih and Chen [4]. However, since then some important contributions also appeared in the literature, e.g. [5], [6].

Of course, anti-plane elastodynamic crack problems are considerably simpler than their counterpart in-plane, since the former involve one wave equation instead of two wave equations for the latter problems. In spite of the idealization of the anti-plane case, this may serve both to examine certain qualitative features common to all types of fracture and to check the efficiency of several numerical methods utilized in fracture mechanics.

Here, we have analyzed an anti-plane crack problem of the transient elastodynamic type. This involves two rapidly propagating cracks in an elastic, isotropic and homogeneous body. Initially, this unbounded body is under a state of constant shear stresses at infinity. At a certain instant of time taken as $t=0$, two cruciform cracks emanate from the same internal point taken as the origin of a Cartesian coordinate system. The cracks propagate along the $x$ and $y$ axes with constant velocities. In particular, the two branches of the first crack on the 
$x$-axis run at different speeds $v_{1}, v_{2}$ which also differ, from the common speed of the branches of the second crack on the $y$-axis $v$. Thus, symmetry prevails only about the $x$-axis.

The present problem is an idealized case of a class of geodynamical fracture problems. Indeed, cruciform crack propagation at different velocities of the several crack branches is common to the fracture of geophysical settings with preexisting rupture planes. It is noted that anti-plane shear crack propagation in geophysical settings with preexisting fracture planes has more physical significance than fracture of engineering materials since in the first case the basic assumptions of the theory are very well fulfilled, viz. nearly infinite thickness of the layer, negligible bending, etc. Of course, the most general case in cruciform crack propagation is the case of four different tip velocities of the respective four crack branches. But such a fully asymmetric problem with unequal crack branches both in $x$ - and $y$-axis would result to an intractable mathematical problem. In this paper, we have confined ourselves to study the effect of asymmetry only in one direction.

Moreover, we have considered that the external stresses at infinity $\tau_{x z}$ and $\tau_{y z}$ are equal for convenience in the computations. Actually, these stresses may be not only different from each other but also arbitrary functions of the spatial variables, without any change in the procedure followed.

The method of solution is based on Chaplygin's transformation and the analytic-function theory. After successive conformal mappings a mixed boundaryvalue problem in the half-plane was formulated and solved.

\section{Governing Equations and Boundary Conditions}

As depicted in Fig. 1, we consider a body occupying the whole space under anti-plane shear at infinity. Assume that this body was disturbed by cruciform cracks running at different velocities along the $x$ - and $y$-axes, respectively. The crack motion produces disturbances such that

$$
u_{x}=u_{y}=0, \quad u_{z}=w(x, y, t)
$$

The $w$-displacement satisfies the two-dimensional wave equation [7]

$$
\nabla 2 w=\frac{1}{c^{2}} \frac{\partial^{2} w}{\partial t^{2}}
$$

where $c=(\mu / \varrho)^{1 / 2}$ is the shear-wave velocity in the medium, $\mu$ the shear modulus and $\varrho$ the mass density of the material.

It is obvious that the time-derivative of the displacement $\dot{w}=\partial w / \partial t$, i.e. the particle velocity, satisfies also the wave equation

$$
\nabla^{2} \dot{w}=\frac{1}{c^{2}} \frac{\partial^{2} \dot{w}}{\partial t^{2}}
$$




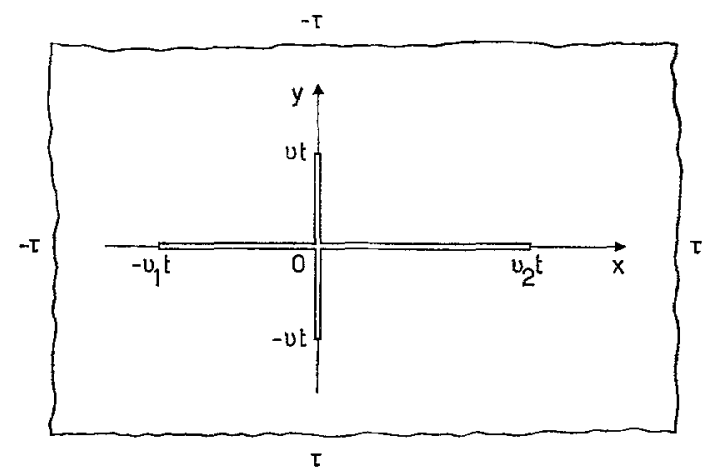

Fig. 1. Crack propagation in asymmetric cruciform paths

which in polar $(r, \theta)$-coordinates may be written as

$$
\frac{\partial^{2} \dot{w}}{\partial r^{2}}+\frac{1}{r} \frac{\partial \dot{w}}{\partial r}+\frac{1}{r^{2}} \frac{\partial^{2} \dot{w}}{\partial \theta^{2}}=\frac{1}{c^{2}} \frac{\partial^{2} \dot{w}}{\partial t^{2}}
$$

The only operative anti-plane shear stresses are given by

$$
\begin{aligned}
& \tau_{x z}=\mu \frac{\partial w}{\partial x}=\mu\left(\cos \theta \frac{\partial w}{\partial r}-\frac{\sin \theta}{r} \frac{\partial w}{\partial \theta}\right) \\
& \tau_{y z}=\mu \frac{\partial w}{\partial y}=\mu\left(\sin \theta \frac{\partial w}{\partial r}+\frac{\cos \theta}{r} \frac{\partial w}{\partial \theta}\right) .
\end{aligned}
$$

For convenience, instead of loading at infinity, we consider the loading as applied to the crack surfaces. Then, the solution of the original problem was extracted by a trivial superposition. Moreover, because of the symmetry in geometry and loading with respect to the plane $y=0$, the problem in the whole space may be reduced in the upper semi-space. In view of the above and Fig. 1, the boundary conditions can then be written as

$$
\begin{array}{lll}
\tau_{y z}(x, 0)=\tau & \text { for } \quad-v_{1} t<x<v_{2} t \\
\tau_{x z}(0, y)=-\tau & \text { for } 0<y<v t \\
w(x, 0)=0 & \text { for } \quad-\infty<x<-v_{1} t, \quad v_{2} t<x<\infty \\
\tau_{y z}=\tau_{x z}=0 & \text { for } \quad\left(x^{2}+y^{2}\right)^{1 / 2} \rightarrow \infty .
\end{array}
$$

The displacement and the particle velocity vanish at the wavefront $r=c t$ since the externally applied stresses and their time-rates do not contain impulse or step functions of time. Therefore, the wavefront $r=c t$ is not a shock (singular) wavefront but $w$ and $\dot{w}$ are continuous functions along it. 


\section{Dynamie Similarity and Conformal Mappings}

Following the technique presented in [8], [9] we introduce a new variable $\omega=r / t$ reducing thus the independent variables from $r, \theta, t$ to $\omega, \theta$. As a consequence, Eq. (4) becomes

$$
\omega^{2}\left(1-\frac{\omega^{2}}{c^{2}}\right) \frac{\partial^{2} \dot{w}}{\partial \omega^{2}}+\omega\left(1-\frac{2 \omega^{2}}{c^{2}}\right) \frac{\partial \dot{w}}{\partial \omega}+\frac{\partial^{2} \dot{w}}{\partial \theta^{2}}=0
$$

in the new "velocity" plane. Accordingly, the distances in the $(x, y)$-physical plane must be transformed to the pertinent variable in the $(x / t, y / t)$-"velocity" plane, as is shown in Fig. 2.

It is advantageous, as it will be seen later, to express the boundary conditions in terms of $\dot{w}$ and $(\partial \dot{w} / \partial \theta)$. In view of (5), Eqs. (6) become

$$
\begin{array}{rlll}
\frac{\partial w}{\partial \theta}=\frac{\tau}{\mu} r & \text { for } & \theta=0, \pi, & -v_{1} t<r<v_{2} t \\
\frac{\partial w}{\partial \theta}=\frac{\tau}{\mu} r & \text { for } \quad \theta=\pi / 2, & & 0<r<v t \\
w=0 & \text { for } \quad \theta=0, \pi, & -v_{1} t>r \quad \text { and } \quad v_{2} i<r .
\end{array}
$$

Differentiation in Eqs. (8) gives automatically the boundary conditions in the "velocity" plane

$$
\begin{array}{rlrlrl}
\frac{\partial \dot{w}}{\partial \theta}=\frac{\tau}{\mu} \omega & \text { for } & \theta=0, \pi, & & -v_{1}<\omega<v_{2} \\
\frac{\partial \dot{w}}{\partial \theta}=\frac{\tau}{\mu} \omega & \text { for } & \theta=\pi / 2, & & 0<\omega<v \\
\dot{w}=0 & \text { for } & \theta=0, \pi, & & -v_{1}>\omega \text { and } v_{2}<\omega \\
\dot{w}=0 & \text { for } & \omega=c, & \pi \leqq \theta \leqq 0 .
\end{array}
$$

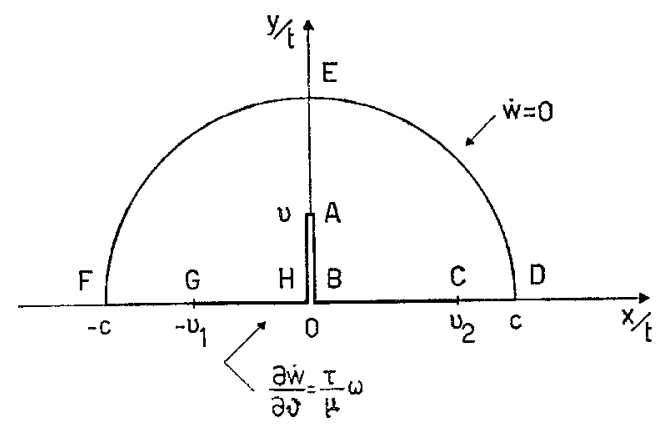

Fig. 2. The "velocity" plane 
The next step is the application of the Chaplygin transformation [8], [9]. According to this, the area inside the half-circle $\omega=c$ with the branch cut along $\theta=\pi / 2,0<\omega<v$ (Fig. 2) is conformally mapped onto the semi-infinite strip $0<s<\infty, 0<\theta<\pi$ with a cut in the $(s, \theta)$-plane (Fig. 3) by means of

$$
\gamma=s+i \theta=\cosh ^{-1}(c / \omega)+i \theta \text {. }
$$

Under (10), Eq. (7) becomes

$$
\frac{\partial^{2} \dot{w}}{\partial s^{2}}+\frac{\partial^{2} \dot{w}}{\partial \theta^{2}}=0
$$

namely the Laplace equation in the $(s, \theta)$-plane. Methods of analytic-function theory are therefore applicable.

It is interesting to observe that $(\hat{\partial} \dot{w} / \partial \theta)$ may be transformed from the $(x / t$, $y / t)$-plane to $(s, \theta)$-plane and vice versa, simply by changing the variable $\omega$ to $s$ via (10). In the $(s, \theta)$-plane, $(\partial \dot{w} / \partial \theta)$ becomes the normal derivative of $\dot{w}(s, \theta)$ along the boundaries $\theta=0, \theta=\pi / 2$ and $\theta=\pi$. The boundary conditions (9) are now expressed by

$$
\begin{aligned}
& \frac{\partial \dot{w}}{\partial \theta}=\frac{\tau}{\mu} \frac{c}{\cosh s} \quad \text { along } \quad G H, A H, A B, C B \\
& \dot{w}=0 \quad \text { along } \quad F G, D E F, C D .
\end{aligned}
$$

The above boundary-value problem has quite unusual boundary conditions. Conditions (12) are not of the well-known types of Dirichlet, Neumann, or RobinCauchy. However, this problem will treat by means of a new conformal mapping of the cut strip onto the upper half-plane $-\infty<\xi<\infty, \eta \geqq 0$ and then by a convenient formulation of a Keldysh-Sedov problem.

We consider the following transformation already utilized in crack problems of elastic wave diffraction by Achenbach [10] and others [11], [12]

$$
\begin{aligned}
& \zeta=\left[1-\left(1-\frac{v^{2}}{c^{2}}\right) \tanh ^{2}(\gamma-i \pi)\right]^{1 / 2} \\
& \gamma=\tanh ^{-1}\left[\left(\frac{1-\zeta^{2}}{1-\frac{v^{2}}{c^{2}}}\right)^{1 / 2}\right]+i \pi
\end{aligned}
$$

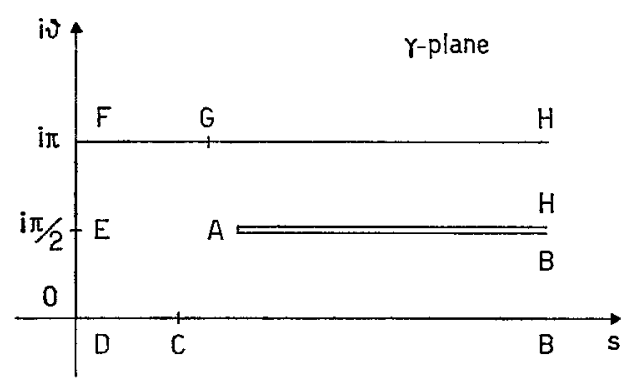

Fig. 3. The semi-infinite strip with a cut in the $(s, \theta)$-plane 
Under the above relations the strip of Fig. 3 maps conformally onto the upper half-plane of Fig. 4. The new positions of the points of interest are also shown in the latter figure.

To transform the boundary conditions from the $(s, \theta)$ - to the $(\xi, \eta)$-plane it must be considered that

$$
\frac{\partial \dot{w}}{\partial \eta}=\frac{d \gamma}{d \zeta} \frac{\partial \dot{w}}{\partial \theta}=\frac{\left[1-(v / c)^{2}\right]^{1 / 2} \zeta}{\left[(v / c)^{2}-\zeta^{2}\right]\left(1-\zeta^{2}\right)^{1 / 2}} \frac{\partial \dot{w}}{\partial \theta} .
$$

Then, the boundary values of the normal derivative $(\partial \dot{w} / \partial \eta)$ are given as

$$
\begin{aligned}
& \frac{\partial \dot{w}}{\partial \eta}=-\frac{\tau c}{\mu} \frac{\xi}{\left[\xi^{2}-(v / c)^{2}\right]^{1 / 2}\left(1-\xi^{2}\right)^{1 / 2}} \quad \text { along } \quad G H, C B \\
& \frac{\partial \dot{w}}{\partial \eta}=\frac{\tau c}{\mu} \frac{\left[1-(v / c)^{2}\right]^{1 / 2} \xi}{\left[(v / c)^{2}-\xi^{2}\right]^{1 / 2}\left(1-\xi^{2}\right)} \quad \text { along } B A, A H .
\end{aligned}
$$

\section{The Koldysh-Sedov Problem}

Since the $\dot{w}(\xi, \eta)$-function satisfies Laplace's equation, we can write

$$
\dot{w}(\xi, \eta)=\operatorname{Re} \phi(\zeta)
$$

where $\phi$ is an analytic function of the complex variable $\zeta=\xi+i \eta$. Consequently, it is valid that

$$
\begin{gathered}
\Phi(\zeta)=\phi^{\prime}(\zeta)=\frac{\partial((\operatorname{Re} \phi(\zeta))}{\partial \xi}+i \frac{\partial(\operatorname{Im} \phi(\zeta))}{\partial \xi} \\
=\frac{\partial(\operatorname{Re} \phi(\zeta))}{\partial \xi}-i \frac{\partial(\operatorname{Re} \phi(\zeta))}{\partial \eta}=\frac{\partial \dot{w}}{\partial \xi}-i \frac{\partial \dot{w}}{\partial \eta} \\
\phi(\zeta)=\int \Phi(\zeta) d \zeta+A,
\end{gathered}
$$

where the constant $A$ may be omitted, since it contributes only a rigid-body motion to the system.

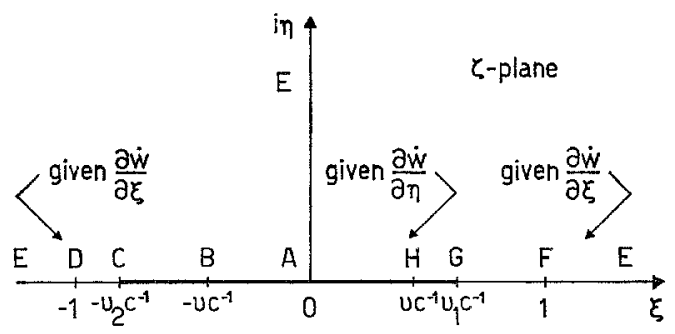

Fig. 4. The upper $(\xi, \eta)$-half plane 
Because $\dot{w}(\xi)$ vanishes in the interval $\xi>v_{1} / c$ and $\xi<-v_{2} / c$ of the real $\xi$-axis, the derivative $(\partial \dot{w} / \partial \xi)$ vanishes also in the same interval. Thus, the original crack problem is reduced to a mixed boundary-value problem where the real part, $\partial \dot{w} / \partial \xi$, of an analytic function $\Phi(\zeta)$ is prescribed on an interval of the real axis of a half-plane, whereas the imaginary part, $\partial \dot{w} / \partial \eta$, is given on the remaining portion of the $\xi$-axis.

One may recognize that this problem is the Keldysh-Sedov mixed-boundary value problem [13], [14]. As regards the type of solution of the problem, we choose a behaviour of the $\phi(\zeta)$-function so that square root singularities of the time-rate of stress occur at $v_{1} / c$ and $-v_{2} / c$. This corresponds to integrable singularities of $\Phi(\zeta)$ at the points of division, i.e. at $v_{1} / c$ and $-v_{2} / c$

$$
\Phi(\zeta)=\frac{1}{R(\zeta)}\left[\frac{1}{2 \pi i} \int_{-\left(v_{\mathbf{2}} / c\right)}^{\left(v_{1} / c\right)} \frac{R\left(\sigma^{\prime}\right) g\left(\sigma^{\prime}\right)}{\sigma-\zeta} d \sigma+B \zeta+C\right]
$$

where

$$
\begin{gathered}
R(\zeta)=\left(\zeta+\frac{v_{2}}{c}\right)^{1 / 2}\left(\zeta-\frac{v_{1}}{c}\right)^{1 / 2} \\
g(\sigma)=-2 i \frac{\partial \dot{w}}{\partial \eta}=\frac{2 \tau c}{\mu} \frac{\sigma}{\left[(v / c)^{2}-\sigma^{2}\right]^{1 / 2}\left(1-\sigma^{2}\right)^{1 / 2}} \quad \text { for } \quad \begin{array}{l}
\left(v_{1} / c\right)>\sigma>(v / c) \quad \text { and } \\
-(v / c)>\sigma>-\left(v_{2} / c\right)
\end{array} \\
=-\frac{i 2 \tau c}{\mu} \frac{\left[1-(v / c)^{2}\right]^{1 / 2} \sigma}{\left[(v / c)^{2}-\sigma^{2}\right]^{1 / 2}\left(1-\sigma^{2}\right)} \text { for } \quad(v / c)>\sigma>-(v / c) .
\end{gathered}
$$

In relation (19) the constant $B$ vanishes due to the obvious additional condition that $\Phi(\infty)=0$. The remaining constant $C$ is a real one.

\section{The Stress Field}

From the view-point of fracture mechanics, the determination of the $\tau_{y z}(x, 0, t)$ and $\tau_{x z}(0, y, t)$-stress is of great importance since the stress intensity factors at the crack tips may then easily be obtained as

$$
\begin{aligned}
& K_{\mathrm{III}}=\lim _{x \rightarrow-v_{1} t}\left[2 \pi\left(x+v_{1} t\right)\right]^{1 / 2} \tau_{y z}(x, 0, t) \\
& K_{\mathrm{III}}=\lim _{x \rightarrow v_{2} t}\left[2 \pi\left(x-v_{2} t\right)\right]^{1 / 2} \tau_{y z}(x, 0, t) \\
& K_{\mathrm{III}}=\lim _{y \rightarrow v t}[2 \pi(y-v t)]^{1 / 2} \tau_{x z}(0, y, t) .
\end{aligned}
$$


Due to the symmetry of the original crack problem in respect to $x$-axis, the determination of the $\tau_{y z}(x, 0, t)$-stress ahead of the moving crack tips does not present a particular interest since it may be obtained by a convenient superposition utilizing the work of Achenbach and Brock [15]. However, the determination of the $\tau_{x z}(0, y, t)$-stress is a novel subject and thus the present analysis is indispensable for the solution of the problem.

In view of relations $(5),(13)$ and $(17)$ the $\dot{\tau}_{x z}(0, y, t)$ time rate of stress may be evaluated as

$$
\begin{aligned}
\dot{\tau}_{x z}(0, y, t) & =\frac{\mu}{y} \operatorname{Im}\left[\Phi(\eta) \frac{d \zeta}{d \gamma}\right], \quad v t<y<c t \\
\frac{d \zeta}{d \gamma} & =\frac{\left[(v / c)^{2}-\zeta^{2}\right]\left(1-\zeta^{2}\right)^{1 / 2}}{\left[1-(v / c)^{2}\right]^{1 / 2} \zeta}
\end{aligned}
$$

and consequently the stress itself as

$$
\tau_{x z}(0, y, t)=\int_{(y / t)}^{t} \dot{\tau}_{x z}(0, y, t) d t, \quad(y / v) \geqq t \geqq(y / c)
$$

Time rates of stress and stress itself are therefore obtained as long as $\Phi(\eta)$ is calculated. Moreover, stresses at any material point can be determined following the evaluation of the $\Phi(\zeta)$-function.

Restricting ourselves to the evaluation of the quantities of interest ahead of the tip moving along the $y$-axis, it suffices to calculate the following integrals

$$
\begin{aligned}
I_{1} & =\int_{(v / c)}^{\left(v_{1} / c\right)}\left[\frac{\left(\sigma+\left(v_{2} / c\right)\right)\left(\sigma-\left(v_{1} / c\right)\right)}{\left((v / c)^{2}-\sigma^{2}\right)\left(1-\sigma^{2}\right)}\right]^{1 / 2} \frac{\sigma d \sigma}{\sigma-i \eta} \\
I_{2} & =\int_{-(v / c)}^{(v / c)}\left[\frac{\left(\sigma+\left(v_{2} / c\right)\right)\left(\sigma-\left(v_{1} / c\right)\right)\left(1-(v / c)^{2}\right)}{(v / c)^{2}-\sigma^{2}}\right]^{1 / 2} \frac{\sigma d \sigma}{(\sigma-i \eta)\left(1-\sigma^{2}\right)} \\
I_{3} & =\int_{-\left(v_{2} / c\right)}^{-(v / c)}\left[\frac{\left(\sigma+\left(v_{2} / c\right)\right)\left(\sigma-\left(v_{1} / c\right)\right)}{\left((v / c)^{2}-\sigma^{2}\right)\left(1-\sigma^{2}\right)}\right]^{1 / 2} \frac{\sigma d \sigma}{\sigma-i \eta} .
\end{aligned}
$$

The $I_{1}$ and $I_{3}$-integrals have the same form which is as follows

$$
I=\int_{\alpha}^{b} f(\lambda)\left(\frac{\lambda-\alpha}{b-\lambda}\right)^{1 / 2} d \lambda
$$


whereas the $I_{2}$-integral is of the form

$$
I^{*}=\int_{a}^{b} f(\lambda)[(\lambda-\alpha)(b-\lambda)]^{-1 / 2} d \lambda .
$$

Both $I$ - and $I^{*}$-integrals are too complicated in our case for a closed-form evaluation. However, one may apply a numerical scheme for their evaluation in order to have useful results for the praxis. For instance, in Abramowitz and Stegun [16] the following numerical formulas are suggested for these integrals

$$
\begin{gathered}
I \cong(b-\alpha) \sum_{j=1}^{n} W_{j} f\left(m_{j}\right) \\
m_{j}=\alpha+(b-\alpha) \lambda_{j} \\
\lambda_{j}=\cos ^{2}\left(\frac{2 j-1}{2 n+1} \frac{\pi}{2}\right) \\
W_{j}=\frac{2 \pi}{2 n+1} \lambda_{j}
\end{gathered}
$$

and

$$
\begin{gathered}
I^{*} \cong \sum_{j=1}^{n} W_{j} f\left(\lambda_{j}\right) \\
\lambda_{j}=\frac{b+\alpha}{2}+\frac{b-\alpha}{2} m_{j} \\
m_{j}=\cos \left(\frac{2 j-1}{2 n} \pi\right) \\
W_{j}=\frac{\pi}{n} .
\end{gathered}
$$

In this way, one may obtain the time-rate of the $\tau_{x z}(0, y, t)$-stress by the relation

$$
\begin{aligned}
\dot{\tau}_{x z}(0, y, t)=\frac{\mu}{y} \operatorname{Im}[ & {\left[( i \eta + \frac { v _ { 2 } } { c } ) ^ { - 1 / 2 } ( i \eta - \frac { v _ { 1 } } { c } ) ^ { - 1 / 2 } \left((2 \pi i)^{-1}\right.\right.} \\
& \left.\left.\cdot\left(2 \tau c \mu^{-1} I_{1}-i 2 \tau c \mu^{-1} I_{2}+2 \tau c \mu^{-1} I_{3}\right)\right)+C\right] \\
& \left.\cdot\left[(v / c)^{2}-(i \eta)^{2}\right]\left[1-(i \eta)^{2}\right]^{1 / 2}\left[1-(v / c)^{2}\right]^{-1 / 2}(i \eta)^{-1}\right]
\end{aligned}
$$

where

$$
i \eta=\left[1-\left(1-\frac{v^{2}}{c^{2}}\right) \tanh ^{2}\left[\cosh ^{-1}\left(\frac{c t}{y /}\right)-\frac{i \pi}{2}\right]\right]^{1 / 2}
$$


The constant $C$ can be determined by the boundary condition $\dot{\tau}_{y z}=0$ at the points $B$ or $H$, i.e. for $\xi=-(v / c)$ or $\xi=v / c$.

A numerical integration again will give the stress via (24).

\section{Conclusions}

In this paper we have analyzed the problem of a rapidly extending cruciform slit under anti-plane shear biaxial loading. The usual assumptions of linear elasticity were considered and the method of dynamic similarity was employed in order to apply complex analysis.

It was shown that a closed form solution is impossible and numerical treatment is thus indispensable. However, the procedure is straightforward and one may easily take information about the field quantities of the problem by using the results of the paper.

\section{References}

[1] Achenbach, J. D.: Dynamic effects in brittle fracture, in: Mechanics today (NematNasser, S., ed.). Pergamon Press 1971.

[2] Achenbach, J. D.: Wave propagation, elastodynamic stress singularities, and fracture, in: Theoretical and applied mechanics (Koiter, W. T., ed.). North-Holland 1976.

[3] Freund, L. B.: The analysis of elastodynamic crack tip stress fields, in: Mechanics today (Nemat-Nasser, S., ed.). Pergamon Press 1974.

[4] Sih, G. C., Chen, E. P.: Cracks moving at constant velocity and acceleration, in: Elastodynamic crack problems (Sih, G. C., ed.). Noordhoff Int. Publ. 1977.

[5] Burgers, P., Freund, L. B.: Dynamic growth of an edge crack in a half space. Int. J. Solids Structures 16, 265-274 (1980).

[6] Brock, L. M., Deng, Y. C.: Dynamic studies of running half- plane and cruciform cracks. Int. J. Engng. Sci. 23, 163-171 (1985).

[7] Achenbach, J. D.: Wave propagation in elastic solids. Amsterdam: North-Holland 1973.

[8] Miles, J. W.: Homogeneous solutions in elastic wave propagation. Quart. Appl. Math. $18,37-59(1960)$.

[9] Craggs, J. W.: On two-dimensional waves in an elastic half-space. Proc. Camb. Phil. Soc. 56, 269-285 (1960).

[10] Achenbach, J. D.: Bifurcation of a running crack in antiplane strain. Int. J. Solids Structures 11, 1301-1314 (1975).

[11] Achenbach, J. D., Varatharajulu, V. K.: Elastodynamic stress-intensity factors for tearing of a half plane. J. Appl. Mech. 41, 1099-1105 (1974).

[12] Burgers, P., Dempsey, J. P.: Two analytical solutions for dynamic crack bifurcation in antiplane straiu. J. Appl. Mech. 49, 366-370 (1982).

[13] Muskhelishvili, N. I.: Singular integral equations. Groningen: Noordhoff 1953.

[14] Gakhov, F. D.: Boundary value problems. New York: Pergamon Press 1966.

[15] Achenbach, J. D., Brock, L. M.: Rapid extension of a crack. J. Elasticity 1, 51-63 (1971). 
[16] Abramowitz, M., Stegun, I. A., (eds.): Handbook of mathematical functions. New York: Dover Publications 1972.

Dr. H. G. Georgiadis

33-35 G. Papandreou Str.

Vironas

GR-16231 Athens

Greece

and

Departement of Mechanical Engineering and Applied Mechanics

The University of Michigan 2250 G. G. Brown Bldg.

Ann Arbor, $M I 48109-2125$

U.S.A. 